\title{
Understanding atrial fibrillation, disease progression, management options, and patient-clinician communication
}

This article was published in the following Dove Press journal:

Patient Intelligence

24 August 201I

Number of times this article has been viewed

\author{
Carrie Nemerovski ${ }^{1,2}$ \\ Jona Lekura ${ }^{2}$ \\ James S Kalus ${ }^{2}$
}

'Eugene Applebaum College of Pharmacy and Health Sciences, Wayne State University, ${ }^{2}$ Department of Pharmacy Services, Henry Ford Hospital, Detroit, MI, USA
Correspondence: James S Kalus Department of Pharmacy Services, Henry Ford Hospital, 2799 West Grand Blvd, Detroit, MI 48202, USA

Tel + I 3139167755

Fax + I 313916 I302

Email jkalusI@hfhs.org

\begin{abstract}
Atrial fibrillation (AF) is the most common cardiac arrhythmia. The progressive and chronic nature of this disease leads to heavy utilization of health care resources, with total health care expenditures estimated to be five times greater for patients with AF compared with those without AF. The management of AF involves thromboembolism prophylaxis, prevention of deterioration of cardiac function, and symptom control. As with other chronic disease states, an essential part of clinical care in AF is increasing a patient's understanding of the pathophysiology of AF, the consequences of untreated AF, and the therapeutic goals and potential side effects of treatment plans. Adherence to treatment regimens has the potential to have a large impact on patient outcomes. A reduction in symptoms along with a decrease in stroke incidence and morbidity may be the result of improved medication adherence. Health care professional communication of the management of AF and the treatment plan to an individual patient is multifaceted and essential to improving patient understanding, adherence to prescribed regimens, and clinical outcomes.
\end{abstract}

Keywords: atrial fibrillation, antiarrhythmic, anticoagulation

\section{Introduction}

Atrial fibrillation (AF) is the most common cardiac arrhythmia and is a growing medical concern. The prevalence of AF increases with age and it occurs in $3.8 \%$ of people 60 years of age and older and in $9.0 \%$ of those 80 years of age and older. ${ }^{1}$ The projected number of people with AF in the US will exceed 10 million by the year $2050 .^{2}$ The progressive and chronic nature of this condition results in increased patient expenditures as well as increased utilization of health care services. It has been reported that the expenditures of annual direct and total costs in patients with $\mathrm{AF}$ are approximately five times as high as those in patients without $\mathrm{AF}^{3}$ Health care resource use and costs attributable to treating this arrhythmia in the US are estimated at $\$ 6.65$ billion annually, including hospitalization, inpatient cost, outpatient treatment, and prescription drugs. ${ }^{4}$

AF is a supraventricular tachyarrhythmia where disorganized electrical impulses originate independent of the sinoatrial node. These impulses result in fibrillation of the atria instead of distinct contractile beats, and lead to an irregular and frequently rapid ventricular response. ${ }^{5}$ In contrast with normal sinus rhythm, the electrocardiogram lacks definite $\mathrm{P}$ waves and the ventricular response, or QRS complex, occurs in an irregularly irregular pattern. AF can be classified by several different systems, but the most commonly used classification is based on temporal patterns and is recommended by the American College of Cardiology/American Heart Association guidelines. ${ }^{5}$ 
Once a patient experiences two or more episodes of AF, it is considered recurrent and can be classified as paroxysmal (recurrent episodes of AF that terminate spontaneously) or persistent (recurrent episodes of AF that are sustained beyond seven days). Persistent AF is considered longstanding if it has lasted for longer than one year and it is considered permanent once attempts at conversion to normal sinus rhythm have failed or have been foregone.

The onset and maintenance of AF is a complex pathophysiologic process that requires both a triggering event and a susceptible physiologic substrate. Once both factors are present, AF may occur, and there are two leading theoretical mechanisms by which AF can be explained, ie, the automatic focus theory and the multiple wavelet triggering hypothesis..$^{5-7}$ The automatic focus theory suggests that the arrhythmia exists only in isolated regions of the heart. Most commonly these foci are seen in the pulmonary vein, but can be present in the atrial myocardium, coronary sinus, superior vena cava, and other cardiac tissues. ${ }^{5}$ The multiple wavelet hypothesis suggests that numerous independent and self-perpetuating wavelets or circuits propagate randomly throughout the atrial myocardium around multiple areas of refractory tissue. Large atrial size coupled with a short refractory period and delayed conduction leads to variable changes in refractoriness and conduction throughout the atria. This combination of factors may provide a milieu for the maintenance of AF. Although debate continues about the role each of these proposed mechanisms plays in the pathogenesis of AF, it is important to realize that these mechanisms are not mutually exclusive and are often present together in the same patient. ${ }^{5-7}$ Regardless of the process, it is clear that AF induces anatomical and physiological distortions throughout the atria through several cellular processes. These structural changes known as "atrial remodeling” help explain the progressive nature of the arrhythmia and give credence to the concept "AF begets AF" which suggests that this tachyarrhythmia perpetuates itself. ${ }^{8}$ Additionally, the renin-angiotensin aldosterone system, inflammation, structural changes associated with aging, the autonomic nervous system, and many other factors likely play a role in the initiation and propagation of $\mathrm{AF}^{8-12}$

In order to control symptoms effectively, prevent deterioration of cardiac function, and reduce morbidity and mortality, the management of AF consists of two main objectives, ie, implement a long-term strategy to prevent cardiac deterioration along with controlling patient symptoms and prevent systemic embolism and stroke. In order to reduce or control patient symptoms and prevent cardiac deterioration, the initial step in AF management involves the selection of either a rate control or rhythm control strategy. The results of several trials ${ }^{13-16}$ concluded that a rate or rhythm control strategy produces similar outcomes in AF. Therefore, a rate control strategy with beta blockers, nondihydropyridine calcium channel blockers, and digoxin is considered a reasonable initial approach to managing AF. Amiodarone can be used as a second-line rate control agent. ${ }^{5}$ However, rhythm control is still a useful strategy in selected patients, and is particularly useful for patients that remain symptomatic despite adequate rate control, or in those patients whose rate cannot be adequately controlled. Younger, symptomatic patients with little underlying heart disease could be considered candidates for a rhythm control strategy as well. When a rhythm control strategy is desired, sinus rhythm is most frequently maintained with antiarrhythmic medications, although serial direct current cardioversion, catheter ablation, or a combination of these therapies may be used. VaughanWilliams class I and III antiarrhythmic agents are useful for treating atrial fibrillation, ${ }^{5}$ although class III agents are used most frequently for AF. ${ }^{17}$

Prevention of thromboembolic events, particularly embolic stroke, is the final goal in the management of AF. During fibrillation, the atria lose the ability to contract fully, leading to stasis of blood which results in clot formation, mainly located in the left atrial appendage. ${ }^{18}$

Embolization of the thrombus can result in stroke, transient ischemic attack, and other clinically significant sequelae. ${ }^{19}$ The risk of stroke can be predicted by a stroke risk index, most commonly the $\mathrm{CHADS}_{2}$ scoring system or the more recently developed $\mathrm{CHA}_{2} \mathrm{DS}_{2}-\mathrm{VAS}_{\mathrm{C}}$ scoring system. ${ }^{20,21}$ These scoring systems are used to quantify the risk of stroke in patients who have AF and help aid in selection of antithrombotic therapy. ${ }^{5,22}$ The risk factors for stroke included in the $\mathrm{CHADS}_{2}$ scoring system are congestive heart failure, hypertension, age $\geq 75$ years, diabetes, and prior cerebral ischemia or transient ischemic attack. ${ }^{20}$ Each risk factor scores one point, except for stroke, which scores two points. In the newer $\mathrm{CHA}_{2} \mathrm{DS}_{2}-\mathrm{VAS}_{\mathrm{C}}$ scoring system, age is weighed differently (age $\geq 75$ years $=2$ points, age 65-74 years $=1$ point), and gender and history of vascular disease are added risk factors. ${ }^{21}$ Warfarin is the preferred antithrombotic medication for AF because it has been found to be more effective than aspirin for prevention of thromboembolism in this patient population. ${ }^{23-26}$ Warfarin is recommended for patients who have a $\mathrm{CHADS}_{2}$ or $\mathrm{CHA}_{2} \mathrm{DS}_{2}-\mathrm{VAS}_{\mathrm{C}}$ score $\geq 2$, whereas aspirin use is advocated in patients with a score of zero. In patients with a score of one, warfarin or 
aspirin can be considered and should be determined based on patient and clinician preference. , 22,27 $^{2}$

In addition to stroke risk assessment, careful assessment of a patient's bleeding risk is also required prior to strategy selection for thromboembolism prophylaxis. Bleeding risk scores, such as the HAS-BLED score, ${ }^{28}$ have been created to help aid the clinician in assessment of bleeding risk. Risk factors that increase a patient's bleeding risk include a history of hypertension, abnormal liver or renal function, stroke, bleeding, labile INRs, age $>65$ years, use of medications that increase bleeding risk, and alcohol abuse. Due to the many similarities between stroke and bleeding risk factors, the utility of the HAS-BLED score in affecting decisions surrounding anticoagulation for individual patients has yet to be elucidated in clinical practice.

In addition to warfarin, other agents are also available for the prevention of thromboembolism in the patient with AF. Based on the findings of The Randomized Evaluation of Long-Term Anticoagulation Therapy (RELY) trial, dabigatran, an oral direct thrombin inhibitor, was found to be superior to warfarin in reducing stroke thromboembolism, with a similar bleeding risk, when given as $150 \mathrm{mg}$ twice daily in patients with $\mathrm{AF}^{29}$ Notably, patients in the $110 \mathrm{mg}$ twice daily arm of RELY experienced a similar risk of thromboembolism and lower risk of bleeding, compared with warfarin. ${ }^{29}$ Both doses are available in Europe and Canada; however, only the $150 \mathrm{mg}$ dose is available in the US. Rivaroxaban and apixaban, both oral Xa antagonists, are also being studied in patients with $\mathrm{AF}^{30,31}$ In the patient who cannot take warfarin, the addition of clopidogrel to aspirin could be an acceptable option, ${ }^{32}$ however, this combination comes with an increased risk of bleeding compared with aspirin alone and is inferior to warfarin for the prevention of stroke. ${ }^{32,33}$

Given the complex nature of the pathophysiology of AF and need for a multifaceted approach to therapy, it is clear that patients with AF must have a strong understanding of their disease state.

\section{Patient understanding of atrial fibrillation}

Although a high level of patient understanding is desirable in the management of AF, it is not always achieved in clinical practice. Several studies have examined the understanding, perceptions, and attitudes that patients have about AF and its treatment. In one study, 119 patients with AF on anticoagulation were surveyed to ascertain their knowledge and perceptions regarding $\mathrm{AF}^{34}$ Only 63\% of patients were aware that their cardiac condition was called AF or fast/irregular heart rate/rhythm/palpitation, and the majority of patients (61\%) felt that their AF was not serious. Only $53 \%$ of patients were aware that AF could predispose them to stroke, and $48 \%$ of patients stated they did not know why they were taking warfarin, but were doing so because their doctor told them to do so. A second study, evaluating an educational intervention to improve patient knowledge related to AF, was conducted in 93 patients with AF on warfarin therapy. ${ }^{35}$ Patients were surveyed prior to the educational intervention and it was reported that only $49 \%$ of patients were aware that their cardiac condition was called AF, but $80 \%$ were aware that $\mathrm{AF}$ is a cardiac rhythm abnormality. Only $41 \%$ of patients felt that AF was a serious condition, and just over half of the patients (53\%) were aware that AF could increase their risk of stroke. Another survey conducted on 100 patients with AF recently discharged from the hospital found that only $46 \%$ of patients recognized that AF was a risk factor for stroke, a mere $25 \%$ of patients were able to identify three or more symptoms to look for related to $\mathrm{AF}$, and only $59 \%$ of patients were able to identify the purpose of their medications used to treat AF. ${ }^{36}$ Lastly, in 2010, the largest survey to date was conducted on 825 patients with AF from 11 different countries. ${ }^{37}$ Although this survey did not directly assess patient knowledge about AF or its treatment, it did assess patient perceptions and attitudes toward this disease state and its management. Among the patients surveyed, almost one quarter of them felt that they would be unable to explain their disease state if asked due to a lack of knowledge, and only $57 \%$ of patients felt that information provided by physicians about AF was easy to understand.

As with other chronic disease states, such as diabetes or heart failure, an essential element to improving patient outcomes in the treatment of AF is a patient's ability to understand their disease state, comprehend their treatment plans, and actively engage in self-care practices. The data presented above suggests that there is room for improvement regarding management of AF between clinicians and patients. AF is a disease that often leads to significant patient morbidity, decreased quality of life, and heavy utilization of health care resources. The approach to reduce this burden on both patients and the health care system for this chronic disease state is no different than other chronic disease states and is rooted in the same concepts of patient engagement, education, and shared decision-making.

Patients diagnosed with AF should understand several key aspects of their disease state and be aware of the reasons for, and side effects of, their medication and treatment plans. Patients need to have a basic understanding of the disease 
state itself. They should know that AF is an abnormal heart rhythm where the upper chambers of the heart do not beat regularly, but instead quiver or shake, causing the heart to beat irregularly and often to beat too quickly. They should understand that AF likely cannot be cured, but can be managed, treated, and controlled. This baseline knowledge of the disease sets the stage for comprehending treatment plans and goals of care.

Once patients understand the pathophysiology of AF they need to be informed of the consequences of untreated AF. To facilitate comprehension and simplify complex medical topics, these consequences can be divided into two broad categories, ie, stroke and deterioration of heart function. First, patients with AF should be informed that they are now at an increased risk of stroke. The concept that the quivering upper chambers of the heart can lead to formation of a blood clot that can ultimately travel to the brain and cause a stroke should be adequately explained to the patient. By effectively teaching this concept to patients, no matter which form of antithrombotic therapy is ultimately chosen, patients may have greater comprehension of the importance of these medications and may be more adherent to stroke prophylaxis regimens. Additionally, patients should be made aware of the signs and symptoms of stroke and advised to seek immediate medical attention if a stroke is suspected.

The second consequence of untreated AF, ie, deterioration of heart function, is a broad concept that encompasses several adverse outcomes that should be explained to all patients. Patients should know that worsening heart function can lead to heart failure, symptoms such as shortness of breath or chest pain, a decreased exercise tolerance, increased hospitalizations, and an overall decrease in their quality of life. With an understanding that the long-term goals of therapy in AF are to prevent worsening cardiac function, patients will be more likely to understand the relevance and importance of their drug therapy regimen.

Once a strategy is selected, further patient education is still needed, but differs based on whether rate or rhythm control is chosen. If rate control is the selected strategy, it should be emphasized to patients that their heart rhythm will remain abnormal or irregular and the purpose of their medication therapy is to control heart rate, in order to reduce the symptoms of AF. Adequately explaining that control of heart rate can prevent or reduce symptoms and prevent the worsening of heart function, will arm patients with the knowledge to understand the treatment plan fully and may influence the patient's adherence to the treatment regimen. Failure to explain the goals of a rate control strategy to patients adequately may lead to miscommunication and frustration on the part of the patient.

If rhythm control is the selected strategy, then an entirely different set of concepts need to be adequately conveyed to the patient. Educational materials detailing the specifics of the method for achieving and maintaining normal sinus rhythm (medications, serial direct current cardioversion, or ablation) will vary depending on which method is ultimately selected. However, the underlying purpose, goals, and expectations of the rhythm control strategy should be explained to all patients. It should be made clear that maintenance of sinus rhythm with any approach is unlikely to keep the patient in sinus rhythm forever. In fact, maintenance of sinus rhythm at one year rarely exceeds $70 \%$ with drug therapy. ${ }^{5}$ Thus, patients should enter into a rhythm control strategy with reasonable expectations. Depending on the patient, the goal may not be to eliminate AF, but rather to reduce the amount of time spent in this abnormal rhythm. Additionally, it should be adequately explained to patients that multiple attempts may be needed to control the abnormal rhythm, and ongoing assessment, monitoring for symptoms, and regular follow-up is essential to achieving the best outcomes. Finally, the adverse effects that could be expected from the antiarrhythmic agent chosen should be explained to the patient. This is important due to the high risk of toxicity with many of the available antiarrhythmic medications.

\section{Patient adherence to treatment}

Patient adherence to medications used in the AF treatment plan can have a major impact on outcomes. Outcomes impacted by anticoagulants, medications for rate control, and medications for rhythm control could all be negatively affected by poor patient adherence. Among patients taking warfarin, it is estimated that patients may be outside of their target INR range approximately $50 \%-60 \%$ of the time. ${ }^{38}$ Nearly $20 \%$ of patients with a low INR were identified as being poorly adherent to their warfarin regimen. ${ }^{39}$ Because poor adherence is a strong contributor to a patient's time spent out of the target INR range, it is clear that there is a strong link between warfarin adherence and potential stroke risk. One important trial demonstrated that risk of stroke increased three-fold when INR decreased below 2. ${ }^{40,41}$ Even when a patient is maintained within the target INR range, stroke can still occur. However, it is notable that when stroke occurs in the setting of a "therapeutic" INR, outcomes of stroke may be less severe and less likely to be fatal. In one study, strokes were less severe or debilitating. ${ }^{42}$ Therefore, patient adherence to an anticoagulant regimen is important for many reasons. 
The most likely reason for a patient with AF to seek medical care and utilize health care resources is the development of symptoms of AF (shortness of breath, palpitations). As noted above, control of heart rate is one of the most effective ways to control symptoms. Therefore, poor adherence to medications used to control heart rate could lead to increased AF symptoms and potentially increased risk of hospitalization. Some heart rate-controlling medications can have bothersome adverse effects, such as exercise intolerance (ie, beta blockers) or constipation (ie, verapamil), which could negatively impact patient adherence. Poor adherence to antiarrhythmic medication regimens may also be common. One study suggests that approximately $30 \%$ of patients were poorly adherent to regimens that consisted primarily of class I antiarrhythmic agents. ${ }^{43}$ Other studies have demonstrated a relatively high rate of discontinuation of antiarrhythmic therapy within one year of initiation. ${ }^{44,45}$ Therefore, the variety of adverse effects that could occur with the different antiarrhythmic agents could be an explanation for this high rate of poor adherence.

\section{Importance of health care professional communication}

There is a great deal of information that must be communicated by health care professionals when caring for a patient with AF. Adequate communication can potentially lead to greater patient satisfaction due to more appropriate expectations, greater adherence to medication regimens, and ultimately could improve patient outcomes. Key points regarding the pathophysiology and consequences of the disease should be communicated upon initial diagnosis of $\mathrm{AF}$ and periodically at follow-up visits. Health care professionals should tell the patient what to expect from the treatment strategy that will be used. For example, if the patient will be treated with a rhythm control strategy, then the health care professional should discuss the potential need for serial electrical cardioversion and hospitalizations if AF recurs. Finally, discussions about medication adverse effects and strategies for avoiding or minimizing adverse effects should be part of any patient visit where a new medication is initiated.

\section{Conclusion}

Ensuring adequate patient understanding of the pathophysiology of AF, the consequences of untreated disease, and the therapeutic goals and potential side effects of treatment is a vital part of caring for a patient with AF. Increased patient knowledge has the potential to increase patient satisfaction with their health care, improve medication adherence, and reduce adverse clinical outcomes. Adequate health care professional communication is the mechanism by which this optimal care can be achieved.

\section{Disclosure}

The authors report no conflicts of interest in this work.

\section{References}

1. Page R. Newly diagnosed atrial fibrillation. N Engl J Med. 2004;351: 2408-2416.

2. Miyasaka Y, Barnes ME, Gersh BJ, et al. Secular trends in incidence of atrial fibrillation in Olmsted County, Minnesota, 1980 to 2000, and implications on the projections for future prevalence. Circulation. 2006;114:119-125.

3. Wu EQ, Birnbaum HG, Mareva M, et al. Economic burden and co-morbidities of atrial fibrillation in a privately insured population. Curr Med Res Opin. 2005;21:1693-1699.

4. Coyne KS, Paramore C, Grandy S, et al. Assessing the direct costs of treating nonvalvular atrial fibrillation in the United States. Value Health. 2006;9:348-356.

5. Fuster V, Ryden LE, Cannom DS, et al. 2011 ACCF/AHA/HRS focused updates incorporated into the ACC/AHA/ESC 2006 guidelines for the management of patients with atrial fibrillation: A Report of the American College of Cardiology Foundation/American Heart Association Task Force on Practice Guidelines. Circulation. 2011;123:e269-e367.

6. Schotten U, Verheule S, Kirchhoff P, Goette A. Pathophysiological mechanisms of atrial fibrillation: A translational appraisal. Physiol Rev. 2011;91:265-325.

7. Nattel S. Atrial electrophysiology and mechanisms of atrial fibrillation. J Cardiovasc Pharmacol Ther. 2003;8 Supp1 1:S5-S11.

8. Wijffels MC, Kirchhof CJ, Dorland R, et al. Atrial fibrillation begets atrial fibrillation. A study in awake chronically instrumented goats. Circulation. 1995;92:1954-1968.

9. Van Wagoner DR. Electrophysiological remodeling in human atrial fibrillation. Pacing Clin Electrophysiol. 2003;26:1572-1575.

10. Iravanian S, Dudley SC Jr. The renin-angiotensin-aldosterone system (RAAS) and cardiac arrhythmias. Heart Rhythm. 2008;5:S12-S17.

11. Zografos T, Katritsis DG. Inhibition of the renin-angiotensin system for prevention of atrial fibrillation. Pacing Clin Electrophysiol. 2010;33:1270-1285.

12. Kalus JS, Coleman CI, White CM. The impact of suppressing the renin-angiotensin system on atrial fibrillation. J Clin Pharmacol. 2006; 46:21-28.

13. Van Gelder IC, Hagens VE, Bosker HA, et al. A comparison of rate control and rhythm control in patients with recurrent persistent atrial fibrillation. N Engl J Med. 2002;347:1834-1840.

14. Wyse DG, Waldo AL, DiMarco JP, et al. A comparison of rate control and rhythm control in patients with atrial fibrillation. $N$ Engl J Med. 2002;347:1825-1833.

15. Opolski G, Torbicki A, Kosior DA, et al. Rate control vs rhythm control in patients with nonvalvular persistent atrial fibrillation: The results of the Polish how to treat chronic atrial fibrillation (HOT CAFÉ) study. Chest. 2004;126:476-486.

16. Hohnloser SH, Kuck KH, Lilienthal J. Rhythm or rate control in atrial fibrillation - pharmacological intervention in atrial fibrillation (PIAF): A randomized trial. Lancet. 2000;356:1789-1794.

17. Fang MC, Stafford RS, Ruskin JN, Singer DE. National trends in antiarrhythmic and antithrombotic medication use in atrial fibrillation. Arch Intern Med. 2004;164:55-60.

18. Blackshear JL, Odell JA. Appendage obliteration to reduce stroke in cardiac surgical patients with atrial fibrillation. Ann Thorac Surg. 1996;61:755-759.

19. Watson T, Shantsila E, Lip GY. Mechanisms of thrombogenesis in atrial fibrillation: Virchow's triad revisited. Lancet. 2009;373:155-166. 
20. Gage BF, Waterman AD, Shannon W, Boechler M, Rich MW, Radford MJ. Validation of clinical classification schemes for predicting stroke. JAMA. 2001;285:2864-2870.

21. Lip GY, Nieuwlaat R, Pisters R, Lane DA, Crijns HJ. Refining clinical risk stratification for predicting stroke and thromboembolism in atrial fibrillation using a novel risk factor-based approach. Chest. 2010;137:263-272.

22. Camm AJ, Kirchhof P, Lip GYH, et al. Guidelines for the management of atrial fibrillation: The task force for the management of atrial fibrillation of the European Society of Cardiology (ESC). Eur Heart J. 2010;31:2369-2429.

23. Stroke Prevention in Atrial Fibrillation Investigators. Stroke Prevention in Atrial Fibrillation Study: Final results. Circulation. 1991;84: 527-539.

24. European Atrial Fibrillation Trial Study Group. Secondary prevention in non-rheumatic atrial fibrillation after transient ischaemic attack or minor stroke. Lancet. 1993;342:1255-1262.

25. Petersen P, Boysen G, Godtfredsen J, et al. Placebo-controlled, randomised trial of warfarin and aspirin for prevention of thromboembolic complications in chronic atrial fibrillation: The Copenhagen AFASAK study. Lancet. 1989;1:175-179.

26. Ezekowitz MD, Bridgers SL, James KE, et al. Warfarin in the prevention of stroke associated with nonrheumatic atrial fibrillation. Veterans Affairs Stroke Prevention in Nonrheumatic Atrial Fibrillation Investigators. N Engl J Med. 1992;327:1406-1412.

27. Singer DE, Albers GW, Dalen JE, et al. Antithrombotic therapy in atrial fibrillation. American College of Chest Physicians Evidence-based Clinical Practice Guidelines (8th ed). Chest. 2008;133:546S-592S.

28. Pisters R, Lane DA, Nieuwlaat R, de Vos CB, Crijns HJ, Lip GY. A novel user friendly score (HAS-BLED) to assess one-year risk of major bleeding in atrial fibrillation patients: The Euro Heart Survey. Chest. 2010;138:1093-1100.

29. Connolly SJ, Ezekowitz MD, Yusuf S, et al. Dabigatran versus warfarin in patients with atrial fibrillation. N Engl J Med. 2009;361:1139-1151.

30. Patel MR, Mahaffey KW, Garg J, et al. Rivaroxaban versus Warfarin in Nonvalvular Atrial Fibrillation. $N$ Engl J Med. Epub 2011, August 10.

31. Connolly SJ, Eikelboom J, Joyner C, et al. Apixaban in patients with atrial fibrillation. $N$ Engl J Med. 2011;364:806-817.

32. Connolly SJ, Pogue J, Hart RG, et al. Effect of clopidogrel added to aspirin in patients with atrial fibrillation. $N$ Engl J Med. 2009;360: 2066-2078.
33. Connolly SJ, Yusuf S, Camm J, et al. Clopidogrel plus aspirin versus oral anticoagulation for atrial fibrillation in the atrial fibrillation clopidogrel trial with irbesartan for prevention of vascular events (ACTIVE W): A randomized controlled trial. Lancet. 2006;367:1903-1912.

34. Lip GYH, Kamath S, Jafri M, Mohammed A, Bareford D, McAlister FA. Ethnic differences in patient perceptions of atrial fibrillation and anticoagulation therapy: The West Birmingham atrial fibrillation project. Stroke. 2002;33:238-242.

35. Lane DA, Ponsford J, Shelley A, Sirpal A, Lip GYH. Patient knowledge and perceptions of atrial fibrillation and anticoagulant therapy: Effects of an educational intervention programme. The West Birmingham atrial fibrillation project. Int J Cardiol. 2006;110:354-358.

36. McCabe PJ, Schad S, Hampton A, Holland DE. Knowledge and self-management behaviors of patients with recently detected atrial fibrillation. Heart Lung. 2008;37:79-90.

37. Aliot E, Breithardt G, Brugada J, et al. An international survey of physician and patient understanding, perception, and attitudes to atrial fibrillation and its contribution to cardiovascular disease morbidity and mortality. Europace. 2010;12:626-633.

38. van Walraven C, Jennings A, Oake N, Fergusson D, Forster AJ. Effect of study setting on anticoagulation control: A systematic review and metaregression. Chest. 2006;129:1155-1166.

39. Rose AJ, Ozonoff A, Grant RW, Henault LE, Hylek EM. Epidemiology of subtherapeutic anticoagulation in the United States. Circ Cardiovasc Qual Outcomes. 2009;2:591-597.

40. Hylek EM, Singer DE. Risk factors for intracranial hemorrhage in outpatients taking warfarin. Ann Intern Med. 1994;120:897-902.

41. Hylek EM, Skates SJ, Sheehan MA, Singer DE. An analysis of the lowest effective intensity of prophylactic anticoagulation for patients with nonrheumatic atrial fibrillation. $N$ Engl J Med. 1996;335:540-546.

42. Hylek EM, Go AS, Chang Y, et al. Effect of intensity of oral anticoagulation on stroke severity and mortality in atrial fibrillation. New Engl J Med. 2003;349:1019-1026.

43. Squire A, Goldman ME, Kupersmith J, Stern EH, Fuster V, Schweitzer P. Long-term antiarrhythmic therapy. Problem of low drug levels and patient noncompliance. Am J Med. 1984;77:1035-1038.

44. Roy D, Talajic M, Dorian P, et al. Amiodarone to prevent recurrence of atrial fibrillation. N Engl J Med. 2000;342:913-920.

45. Singh BN, Singh SN, Reda DJ, et al. Amiodarone versus sotalol for atrial fibrillation. $N$ Engl J Med. 2005;352:1861-1872.
Patient Intelligence

\section{Publish your work in this journal}

Patient Intelligence is an international, peer-reviewed, open access journal that characterizes and measures the central role of patient behavior and intention in optimizing healthcare management in all areas of disease and complaint types. An improved understanding of patient intelligence coupled with predictive analysis helps an organization contribute more effectively to achieving better outcomes. Submit your manuscript here: http://www.dovepress.com/patient-intelligence-journal

\section{Dovepress}

The journal is characterized by the rapid reporting of reviews, original research, methodologies, analytics, modeling, clinical studies and patient surveys across all disease areas. The manuscript management system is completely online and includes a very quick and fair peer-review system. Visit http://www.dovepress.com/ testimonials.php to read real quotes from published authors. 